Prof. Dr. med. Matthias Blumenstein

Ärztlicher Direktor

Klinik Augustinum München

\section{Vorhofflimmern}

25-Diagnostik

27 - Therapie

31 - CME-Fragen

\title{
Antikoagulation bei Vorhofflimmern
}

\section{Zwei von drei Schlaganfällen verhindert}

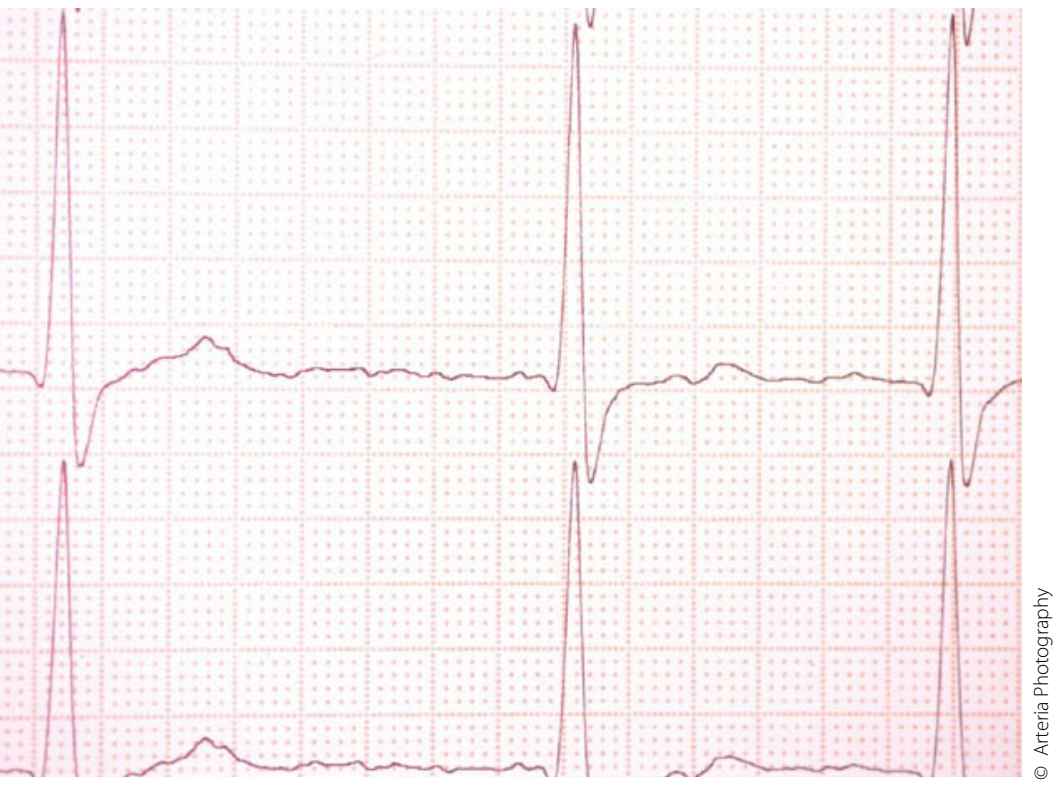

— Vorhofflimmern ist die häufigste Rhythmusstörung des Herzens in der klinischen Praxis. Mit zunehmendem Alter steigt die Häufigkeit an, sodass bei Patienten über 65 Jahren von einer Prävalenz von 6-10\% auszugehen ist.

Vorhofflimmern ist mit einer erheblichen Morbidität und Mortalität verbunden. Es sind weniger kardiale Probleme wie die Entwicklung einer Herzinsuffizienz als thrombembolische Komplikationen, die Anlass zu häufigen Krankenhauseinweisungen geben. Über $80 \%$ hiervon betreffen das Gehirn in Form ischämischer Insulte. Bei Patienten mit einem rheumatischen Klappenvitium und Vorhofflimmern besteht ein bis zu 17-faches Embolierisiko. Aber auch bei nicht valvulärem Vorhofflimmern liegt das Risiko für einen zerebralen Insult immerhin bei 4-6\% pro Jahr.

Neben dem Alter sind für das individuelle thrombembolische Risiko eines Patienten mit Vorhofflimmern Risikofaktoren einer begleitend vorliegenden Atherosklerose bedeutungsvoll. Darüber hinaus erhöhen auch Erkrankungen wie beispielsweise eine klinisch manifeste
Herzinsuffizienz, ein vorangegangener Schlaganfall, eine arterielle Hypertonie oder ein Diabetes mellitus die Wahrscheinlichkeit ischämischer Komplikationen signifikant.

Diese Informationen untermauern, dass neben der primären Behandlung einer Herzrhythmusstörung der antithrombotischen Therapie eine herausragende Bedeutung eingeräumt werden muss, wenn die schlechte Prognose verbessert werden soll.

Der Effekt einer antithombotischen Therapie ist heute gesichert. Dies gilt insbesondere für die orale Antikoagulation mit VitaminK-Antagonisten. In großen Untersuchungen wurde gezeigt, dass bei kontrolliertem Einsatz eine absolute Risikoreduktion für Schlaganfälle von über $60 \%$ gegenüber Placebo erreicht werden kann. Im Vergleich hierzu sind die Ergebnisse einer oralen Thrombozytenaggregationshemmung mit den heute zur Verfügung stehenden Substanzklassen deutlich schlechter. Sie spielt deshalb in aktuellen Therapierichtlinien für Risikopatienten keine Rolle.

\section{Zu viele Patienten schlecht eingestellt}

Kritisch ist anzumerken, dass die orale Antikoagulation mit Vitamin-K-Antagonisten durchaus nicht problemlos ist. In klinischen Studien fanden sich bei bis zu einem Drittel der Patienten INR-Werte außerhalb des vorgegebenen Zielbereichs. Von weit höheren Zahlen schlecht kontrollierter INR-Werte ist im ambulanten Praxisalltag auszugehen. Zudem kommt es vor, dass Patienten bei der Entlassung aus stationärer Behandlung trotz des Vorliegens einer thrombembolischen Risikokonstellation keine eindeutigen Therapieempfehlungen erhalten. Diese Unzulänglichkeiten haben zwangsläufig entweder mangelnde Therapieeffizienz im Falle der Unterdosierung oder aber die Gefahr von Blutungskomplikationen bei Überdosierung der Medikation zur Folge. Entsprechend groß sind die Vorbehalte auf Seiten der Patienten, nicht selten auch der behandelnden Ärzte. Neue pharmakologische Therapieempfehlungen der Fachgesellschaften sollten dazu beitragen, diese Unzulänglichkeiten auszugleichen. Hierzu möchte ich Ihnen das Studium der nachfolgenden Schwerpunktbeiträge wärmstens ans Herz legen. 